\title{
Measurement of Coupling PDC photon sources with single-mode and multimode optical fibers
}

\author{
Stefania Castelletto ${ }^{b}$, Ivo Pietro Degiovanni ${ }^{b}$, Alan Migdall ${ }^{a}$, Valentina Schettini ${ }^{b}$, Michael \\ Ware $^{a}$ \\ ${ }^{a}$ Optical Technology Division, \\ National Institute of Standards and Technology,Gaithersburg, Maryland 20899-8441, \\ ${ }^{b}$ Istituto Elettrotecnico Nazionale G. Ferraris, Strada delle Cacce 91-10135 Torino (Italy)
}

\begin{abstract}
We investigate the coupling efficiency of parametric downconversion light (PDC) into single and multi-mode optical fibers as a function of the pump beam diameter, crystal length and walk-off. We outline two different theoretical models for the preparation and collection of either single-mode or multi-mode PDC light (defined by, for instance, multi-mode fibers or apertures, corresponding to bucket detection). Moreover, we define the mode-matching collection efficiency, important for realizing a single-photon source based on PDC output into a well-defined single spatial mode. We also define a multimode collection efficiency that is useful for single-photon detector calibration applications.
\end{abstract}

\section{INTRODUCTION}

Earliest studies of parametric downconversion (PDC) addressed problems in fundamental physics, while more recent studies target applications such as quantum metrology ${ }^{1}$ and quantum information. ${ }^{2,3}$ While both of these areas make use of two-photon light, they are distinct applications that present different requirements for that light. The stringent requirements of these applications are driving researchers to optimize the PDC process. For these efforts to succeed, a clear theoretical framework is needed.

PDC produces a quantum state of light with a two-photon field description. However, if only one photon of the pair is measured, the source exhibits purely thermal statistical behavior, given by intrinsic multi-mode photon production. We can however introduce a certain degree of coherence. By measuring one of the photons, we prepare the other photon in a specific state. The prepared state will be pure only if we project the first PDC photon (called also the heralding photon) into a pure state. In each of the above PDC applications, we prepare one photon by measuring its twin. Thus for optimization of the process, it is crucial to have a proper definition and measurement of the efficiency of that preparation and of the related mode-matching. We present two different models to define and optimize the two-photon-mode preparation and mode-matching efficiencies. They are distinguished by how the heralding photon is collected. One uses a multimode spatial filter or a bucket detection system, while the second uses a single-mode fiber. A bucket detector is a multimode detector where all the modes are detected jointly. Hence the information about the location of the detected photon (or equivalently in what mode the photons were detected) is "erased". We obtain different dependencies of the efficiency on the pump parameters in these two arrangements. This is particularly important for two specific applications: the calibration of a single-photon detector and the realization of a single-photon on demand source (SPOD). ${ }^{4,5}$

\section{BIPHOTON FIELD}

We consider a two-photon wavefunction, written as ${ }^{6}$

$$
|\psi\rangle=\int \mathrm{d}^{2} \rho_{1} \mathrm{~d}^{2} \rho_{2} \mathrm{~d} t_{1} \mathrm{~d} t_{2} \widetilde{\Phi}\left(\rho_{1}, \rho_{2}, t_{1}, t_{2}\right)\left|1_{\rho_{2}, t_{2}}\right\rangle\left|1_{\rho_{1}, t_{1}}\right\rangle
$$

where $\rho_{1,2}$ represents the transverse positions of the two photons at the instant $t_{1,2}$ and $\widetilde{\Phi}\left(\rho_{1}, \rho_{2}, t_{1}, t_{2}\right)$ is the biphoton field. Its calculation is analytic only for first-order approximation of the transverse wavevectors

Further author information: (Send correspondence to Stefania Castelletto)

E-mail: castelle@ien.it, Telephone: 00390113919223 
(assuming the pump, signal, and idler have narrow transverse angular distributions we can adopt the paraxial approximation), and is obtained by performing the Fourier transform of the pump angular distribution and the phase-matching function with respect to the pump transverse k-components and the signal k-components. Perfect transverse phase-matching is also assumed. The result derived in ref. ${ }^{7}$ is

$$
\begin{aligned}
\widetilde{\Phi}\left(\rho_{1}, \rho_{2}, t_{1}, t_{2}\right)= & N_{1} \exp \left[\frac{-i\left(K_{i} \theta_{i}^{2}+K_{s} \theta_{s} \theta_{i} \tau\right)}{D}\right] \exp \left[\frac{-\left(\mathcal{N}_{p}-\mathcal{N}_{s}\right)^{2} \tau^{2}}{D^{2} w_{p}^{2} K_{p}}\right] \\
& \times \exp \left[\frac{2\left(\mathcal{N}_{p}-\mathcal{N}_{s}\right) \tau\left(y_{1}+\frac{\theta_{i} \tau}{D}\right)}{D w_{p}^{2} K_{p}}\right] \exp \left[-\frac{x_{1}^{2}+\left(y_{1}+\frac{\theta_{i} \tau}{D}\right)^{2}}{w_{p}^{2}}\right] \Pi_{D L}(\tau) \\
& \times \delta\left(x_{1}-x_{2}\right) \delta\left(y_{1}-y_{2}+\frac{\left(\theta_{i}+\theta_{s}\right) \tau}{D}\right),
\end{aligned}
$$

where $\tau=t_{1}-t_{2}$ and $\Pi_{D L}(\tau)=1$ for $0 \leq \tau \leq D L$ and 0 elsewhere. The subscripts $s, i, p$ indicate the signal, idler, and pump. $\theta_{i, s}$ are the central emission angles (in a small angle non-collinear approximation), and $K_{i, s, p}=n_{i, s, p}\left(\Omega_{i, s, p}, \phi\right) \Omega_{i, s, p} / c$ describe the directions of the central intensities of the wavevectors. The terms, $\mathcal{N}_{p}=\left.\frac{\Omega_{p}}{c} \frac{\mathrm{d} n_{p}\left(\Omega_{p}, \phi\right)}{\mathrm{d} \phi}\right|_{\phi_{o}}$ and $\mathcal{N}_{s}=\left.\frac{\Omega_{s}}{c} \frac{\mathrm{d} n_{s}\left(\Omega_{s}, \phi\right)}{\mathrm{d} \phi}\right|_{\phi_{o}}$ account for the effects on the refractive indexes $\left(n_{p, s, i}\left(\omega_{p, s, i}, \phi\right)\right.$ expanded around the central frequencies $\left(\Omega_{s, i}\right)$, and around the phase-matching angle $\left.\phi_{o}\right)$ of the pump and the signal due to the pump angular spread, which are responsible for a small deviation from the phase-matching angle $\phi_{o} . \quad D=-\left.\frac{\mathrm{d} n_{\mathrm{i}}\left(\omega_{\mathrm{i}}\right) \omega_{\mathrm{i}} / c}{\mathrm{~d} \omega_{\mathrm{i}}}\right|_{\Omega_{\mathrm{i}}}+\left.\frac{\mathrm{d} n_{\mathrm{s}}\left(\omega_{\mathrm{s}}, \phi\right) \omega_{\mathrm{s}} / c}{\mathrm{~d} \omega_{\mathrm{s}}}\right|_{\Omega_{\mathrm{s}}}$ is the differential phase velocity between the signal and idler photons in the crystal. $D=0$ and $\mathcal{N}_{s}=0$ for type I degenerate phase-matching. The pump-beam transverse field distribution is assumed to be Gaussian with a waist of $w_{\mathrm{p}}$ at the crystal. We also assume that the pump propagates with negligible diffraction inside the crystal. In the following, the idler and signal space-time positions are indicated by 1 and 2 , respectively.

\section{SINGLE MODE EFFICIENCY}

Envisioning the optics setup as the unfolded scheme of Fig. $1,{ }^{8}$ the source is described by the propagation of a coherent mode defined by $\varphi_{l m}\left(\rho_{3}\right)$ through an optical element with impulse response function $h_{i}\left(\rho_{3}, \rho_{1}\right)$, through the non-linear crystal where the mode gets transformed according to the phase matching function $\widetilde{\Phi}\left(\rho_{1}, \rho_{2}, t_{1}, t_{2}\right)$, and collected eventually by $h_{s}\left(\rho_{2}, \rho_{4}\right)$. The actual collected mode will then be given by the field $\varphi_{l m}\left(\rho_{4}\right)$.

The coincidences measured at the positions 3 and 4 are then $\mathcal{C}_{34}$, the overlap between the PDC field and both of the preparation or collection modes, while the single counts $\mathcal{C}_{3}$ and $\mathcal{C}_{4}$ measure the overlap individually between the biphoton field and each of the preparing or collecting modes:

$$
\begin{aligned}
\mathcal{C}_{34}= & \int \mathrm{d} t_{1} \mathrm{~d} t_{2} \\
& \left|\int \mathrm{d}^{2} \rho_{1} \mathrm{~d}^{2} \rho_{2} \mathrm{~d}^{2} \rho_{3} \mathrm{~d}^{2} \rho_{4} \widetilde{\Phi}\left(\rho_{1}, t_{1}, \rho_{2}, t_{2}\right) h_{i}\left(\rho_{1}, \rho_{3}\right) h_{s}\left(\rho_{2}, \rho_{4}\right) \varphi_{l^{\prime} m^{\prime}}^{*}\left(\rho_{4}\right) \varphi_{l m}^{*}\left(\rho_{3}\right)\right|^{2} \\
\mathcal{C}_{3}= & \int \mathrm{d} t_{1} \mathrm{~d} t_{2} \mathrm{~d}^{2} \rho_{4}\left|\mathrm{~d}^{2} \rho_{1} \mathrm{~d}^{2} \rho_{2} \mathrm{~d}^{2} \rho_{3} \widetilde{\Phi}\left(\rho_{1}, t_{1}, \rho_{2}, t_{2}\right) h_{i}\left(\rho_{1}, \rho_{3}\right) h_{s}\left(\rho_{2}, \rho_{4}\right) \varphi_{l m}^{*}\left(\rho_{3}\right)\right|^{2} \\
\mathcal{C}_{4}= & \int \mathrm{d} t_{1} \mathrm{~d} t_{2} \mathrm{~d}^{2} \rho_{3}\left|\mathrm{~d}^{2} \rho_{1} \mathrm{~d}^{2} \rho_{2} \mathrm{~d}^{2} \rho_{3} \widetilde{\Phi}\left(\rho_{1}, t_{1}, \rho_{2}, t_{2}\right) h_{i}\left(\rho_{1}, \rho_{3}\right) h_{s}\left(\rho_{2}, \rho_{4}\right) \varphi_{l m}^{*}\left(\rho_{4}\right)\right|^{2}
\end{aligned}
$$

Here we distinguish between the single-mode matching efficiency calculated by

$$
\chi_{M}=\frac{\mathcal{C}_{34}}{\sqrt{\mathcal{C}_{3} \mathcal{C}_{4}}},
$$

and the single-mode preparation efficiency

$$
\chi_{P}{ }^{(3,4)}=\frac{\mathcal{C}_{34}}{\mathcal{C}_{3,4}} .
$$




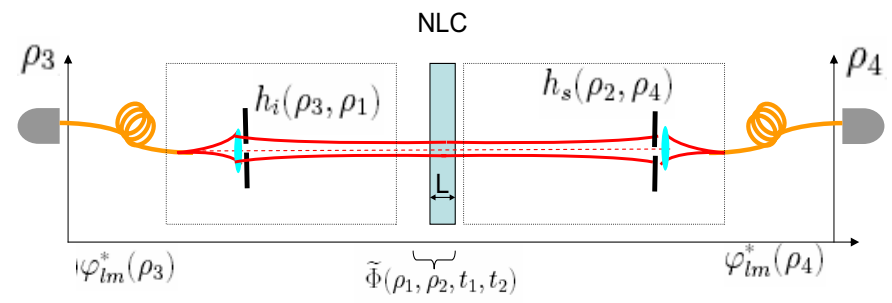

Figure 1. Unfolded picture of an object corresponding to the idler channel of a PDC source, generated in a nonlinear crystal NLC of length $L$. The object (on the left) is a coherent source when the idler beam is prepared by single-mode fiber.

Eq.(4) is the appropriate efficiency to optimize when the final goal is a single photon source on demand emitted in defined photon modes, while Eq.(5) is the efficiency measured when single photon detector calibration is required. Specifically in the paper, we calculate the efficiency in a perfect imaging configuration, namely $h_{s}\left(\rho_{2}, \rho_{4}\right)=$ $\delta\left(\rho_{2}-M_{4} \rho_{4}\right)$ and $h_{i}\left(\rho_{3}, \rho_{1}\right)=\delta\left(\rho_{1}-M_{3} \rho_{3}\right)$ (lenses have infinite aperture with magnification $\left.M_{j+2}\right)$. The lenses are arranged to place the preparation and collection beam waists, $w_{o, j}=250 \mu \mathrm{m}$ at the crystal, with guided Gaussian field modes $\varphi_{10}\left(\rho_{j+2}\right)=\sqrt{\frac{2}{\pi}} \frac{M_{j+2}}{w_{o, j}} \exp \left[-\frac{\rho_{j+2}^{2} M_{j+2}^{2}}{w_{o, j}^{2}}\right]$, with $j=1,2$. The spatial coherence of the single guided modes in the signal and idler arms should ultimately match the overall spatial coherence of the two-photon states. By explicit calculation the single-mode matching efficiency is then given by

$$
\chi_{M}=\mathcal{F}_{M} \frac{4 w_{o, 1}^{2} w_{o, 2}^{2} w_{p}^{2} \sqrt{A}}{\sqrt{\left(w_{o, 2}^{2} w_{p}^{2}+w_{o, 1}^{2}\left(w_{o, 2}^{2}+w_{p}^{2}\right)\right)^{3} B}}
$$

with $A=\left(-\mathcal{N}_{p}+\mathcal{N}_{s}+K_{p} \theta_{i}\right)\left(\mathcal{N}_{p}-\mathcal{N}_{s}+K_{p} \theta_{s}\right) \sqrt{w_{o, 1}^{2}+w_{p}^{2}} \sqrt{w_{o, 2}^{2}+w_{p}^{2}}$,

$$
\mathcal{F}_{M}=\frac{\operatorname{Erf}\left[\frac{L \sqrt{2 B}}{K_{p} \sqrt{w_{o, 2}^{2} w_{p}^{2}+w_{o, 1}^{2}\left(w_{p}^{2}+w_{o, 2}^{2}\right)}}\right]}{\sqrt{\operatorname{Erf}\left[\sqrt{2} L \frac{\left(-\mathcal{N}_{p}+\mathcal{N}_{s}+K_{p} \theta_{i}\right)}{K_{p} \sqrt{w_{o, 1}^{2}+w_{p}^{2}}}\right] \operatorname{Erf}\left[\sqrt{2} L \frac{\left(\mathcal{N}_{p}-\mathcal{N}_{s}+K_{p} \theta_{s}\right)}{K_{p} \sqrt{w_{o, 2}^{2}+w_{p}^{2}}}\right]}}
$$

and $B=\left(\mathcal{N}_{p}-\mathcal{N}_{s}+K_{p} \theta_{s}\right)^{2} w_{o, 1}^{2}+\left(-\mathcal{N}_{p}+\mathcal{N}_{s}+K_{p} \theta_{i}\right)^{2} w_{o, 2}^{2}+K_{p}^{2}\left(\theta_{i}+\theta_{s}\right)^{2} w_{p}^{2}$. In the thin crystal limit this reduces to

$$
\chi_{M}=\frac{4 w_{p}^{2} w_{o, 1}^{2} w_{o, 2}^{2} \sqrt{\left(w_{o, 1}^{2}+w_{p}^{2}\right)\left(w_{o, 2}^{2}+w_{p}^{2}\right)}}{\left(w_{o, 2}^{2} w_{p}^{2}+w_{o, 1}^{2}\left(w_{o, 2}^{2}+w_{p}^{2}\right)\right)^{2}}
$$


as first calculated in Refs.. ${ }^{9,10}$ The single mode preparation efficiency is given by

$$
\chi_{P}^{(3,4)}=\mathcal{F}_{P} \frac{4\left(-\mathcal{N}_{p}+\mathcal{N}_{s}+K_{p} \theta_{i}\right) w_{o, 1}^{2} w_{o, 2}^{2} w_{p}^{2} \sqrt{w_{o, 1}^{2}+w_{p}^{2}}}{\sqrt{\left(w_{o, 2}^{2} w_{p}^{2}+w_{o, 1}^{2}\left(w_{o, 2}^{2}+w_{p}^{2}\right)\right)^{3} B}}
$$

with

$$
\mathcal{F}_{P}=\frac{\operatorname{Erf}\left[\frac{L \sqrt{2 B}}{K_{p} \sqrt{w_{o, 2}^{2} w_{p}^{2}+w_{o, 1}^{2}\left(w_{p}^{2}+w_{o, 2}^{2}\right)}}\right]}{\operatorname{Erf}\left[\sqrt{2} L \frac{\left(-\mathcal{N}_{p}+\mathcal{N}_{s}+K_{p} \theta_{i}\right)}{K_{p} \sqrt{w_{o, 1}^{2}+w_{p}^{2}}}\right]}
$$

Also in this case we can calculate easily the thin crystal limit

$$
\chi_{P}=\frac{4 w_{p}^{2} w_{o, 1}^{2} w_{o, 2}^{2}\left(w_{o, 1}^{2}+w_{p}^{2}\right)}{\left(w_{o, 2}^{2} w_{p}^{2}+w_{o, 1}^{2}\left(w_{o, 2}^{2}+w_{p}^{2}\right)\right)^{2}}
$$

Note that

$$
\chi_{M}=\sqrt{\chi_{P}^{(3)} \chi_{P}^{(4)}}
$$

In this case the information of the location of the prepared and detected modes is included in the measurement. In Fig.2(a), we plot the theoretical prediction of $\chi_{P}$ for a $5 \mathrm{~mm}$ crystal of $\mathrm{LiIO}_{3}$ versus the collecting waist $w_{o, 2}$ for fixed preparing waist $w_{o, 1}=250 \mu \mathrm{m}$ at the crystal, for different pump waist configurations. As it appears there is an optimum collecting waist and the best efficiency is obtained for the larger pump waist. $\chi_{M}$ behaves as $\chi_{P}$, except that the highest efficiency is obtained when the preparing and collecting waist are the same $\left(w_{o, 1}=w_{o, 2}\right)$. This can be clearly observed in Fig.2(b)where the optimum collecting waist is plotted versus the pump waist for different prepared waist $w_{o, 1}$. The optimum $w_{o, 2}$ (giving the highest efficiency) approaches $w_{o, 1}$ as the pump waist increases, and this approach is faster in the $\chi_{M}$ case, as expected.

\section{MULTI-MODE OR BUCKET DETECTION EFFICIENCY}

In the case of multi-mode or bucket detection efficiency the schematic is Fig. 3, where we overlap the PDC wave function with the impulse response function of the optical system, and eventually we average over the spatial distribution of multi-mode fibers or spatial filters (apertures) or bucket detector, erasing the information about the spatial location where the photons where collected. More specifically, in Fig. 3, the source is considered incoherent with finite transverse distribution $\mathcal{T}_{3}\left(\rho_{3}\right)$. The collecting modes are then represented by the spatial filter and described by $\mathcal{T}_{4}\left(\rho_{4}\right)$. The coincidences measured at the positions 3 and 4 are then $C_{34}$, and $C_{3}$ and $C_{4}$ are the single counts:

$$
\begin{aligned}
C_{34}= & \int \mathrm{d} t_{1} \mathrm{~d} t_{2} \mathrm{~d}^{2} \rho_{3} \mathrm{~d}^{2} \rho_{4} \mathcal{T}_{4}\left(\rho_{4}\right) \mathcal{T}_{3}\left(\rho_{3}\right) \\
& \left|\int \mathrm{d}^{2} \rho_{1} \mathrm{~d}^{2} \rho_{2} \widetilde{\Phi}\left(\rho_{1}, t_{1}, \rho_{2}, t_{2}\right) h_{i}\left(\rho_{1}, \rho_{3}\right) h_{s}\left(\rho_{2}, \rho_{4}\right)\right|^{2} \\
C_{3}= & \int \mathrm{d} t_{1} \mathrm{~d} t_{2} \mathrm{~d}^{2} \rho_{4} \mathrm{~d}^{2} \rho_{3} \mathcal{T}_{3}\left(\rho_{3}\right)\left|\mathrm{d}^{2} \rho_{1} \mathrm{~d}^{2} \rho_{2} \widetilde{\Phi}\left(\rho_{1}, t_{1}, \rho_{2}, t_{2}\right) h_{i}\left(\rho_{1}, \rho_{3}\right) h_{s}\left(\rho_{2}, \rho_{4}\right)\right|^{2} \\
C_{4}= & \int \mathrm{d} t_{1} \mathrm{~d} t_{2} \mathrm{~d}^{2} \rho_{3} \mathrm{~d}^{2} \rho_{4} \mathcal{T}_{4}\left(\rho_{4}\right)\left|\mathrm{d}^{2} \rho_{1} \mathrm{~d}^{2} \rho_{2} \widetilde{\Phi}\left(\rho_{1}, t_{1}, \rho_{2}, t_{2}\right) h_{i}\left(\rho_{1}, \rho_{3}\right) h_{s}\left(\rho_{2}, \rho_{4}\right)\right|^{2}
\end{aligned}
$$

The 2-photon multi-mode matching efficiency is then defined by ${ }^{11}$

$$
\eta_{M}=\frac{C_{34}}{\sqrt{C_{3} C_{4}}} .
$$

and the 2-photon multi-mode preparation efficiency is defined by

$$
\eta_{P}^{(3,4)}=\frac{C_{34}}{C_{3,4}}
$$


(a)

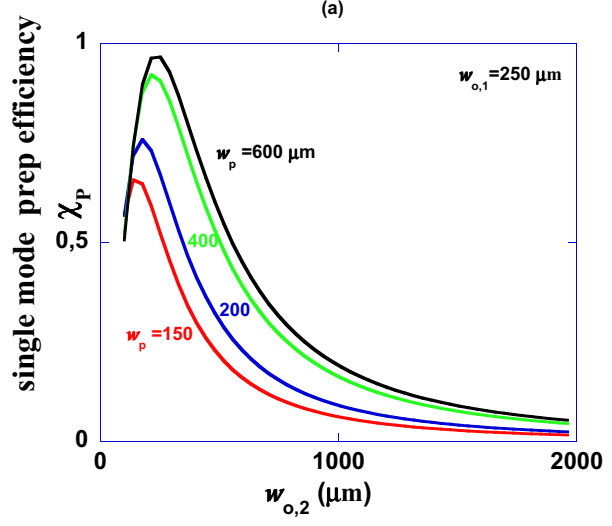

(b)

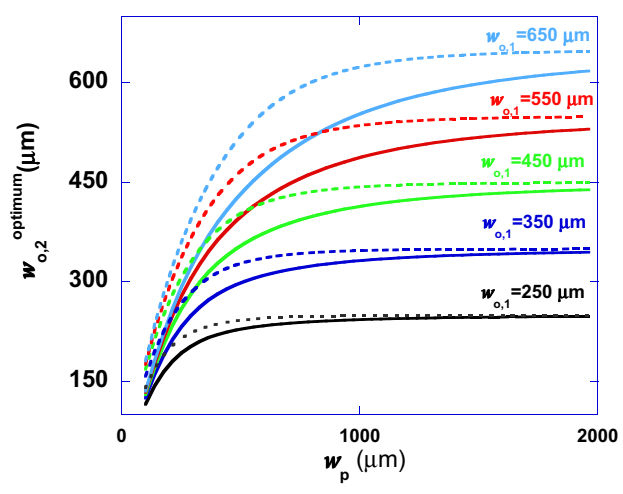

Figure 2. (a) Plot of $\chi_{P}$ versus the collecting mode waist for various pump waist $w_{p}=150,200,400,600 \mu \mathrm{m}$ at $w_{o, 1}=250$ $\mu \mathrm{m}$ (b) Plot of the optimum waist $w_{o, 2}$ versus the pump waist for various preparing waists $w_{o, 1}$ in the case of $\chi_{M}$ (dashed line) and $\chi_{P}$ (solid line).

We calculate the multi-mode matching and preparation efficiencies, assuming the incoherent source is completely determined by the functions $\mathcal{T}_{j+2}\left(\rho_{j+2}\right)=e^{-\frac{2 \rho_{j+2}^{2} M_{j+2}^{2}}{w_{o, j}^{2}}}$, where the impulse response function is the Dirac delta as before. Calculating the multi-mode matching efficiency we obtain:

$$
\eta_{M}=\mathcal{F}_{M} \frac{w_{o, 1} w_{o, 2} \sqrt{A}}{\sqrt{\left(w_{o, 2}^{2} w_{p}^{2}+w_{o, 1}^{2}\left(w_{o, 2}^{2}+w_{p}^{2}\right)\right) B}}
$$

In the thin crystal limit $\eta_{M}$ becomes

$$
\eta_{M}=\frac{w_{o, 1}^{2} w_{o, 2}^{2} \sqrt{\left(w_{o, 1}^{2}+w_{p}^{2}\right)\left(w_{o, 2}^{2}+w_{p}^{2}\right)}}{w_{o, 2}^{2} w_{p}^{2}+w_{o, 1}^{2}\left(w_{o, 2}^{2}+w_{p}^{2}\right)}
$$

Also in this case the multi mode preparation efficiency is given by

$$
\eta_{P}^{(3,4)}=\mathcal{F}_{P} \frac{4\left(-\mathcal{N}_{p}+\mathcal{N}_{s}+K_{p} \theta_{i}\right) w_{o, 2}^{2} \sqrt{w_{o, 1}^{2}+w_{p}^{2}}}{\sqrt{\left(w_{o, 2}^{2} w_{p}^{2}+w_{o, 1}^{2}\left(w_{o, 2}^{2}+w_{p}^{2}\right)\right) B}},
$$




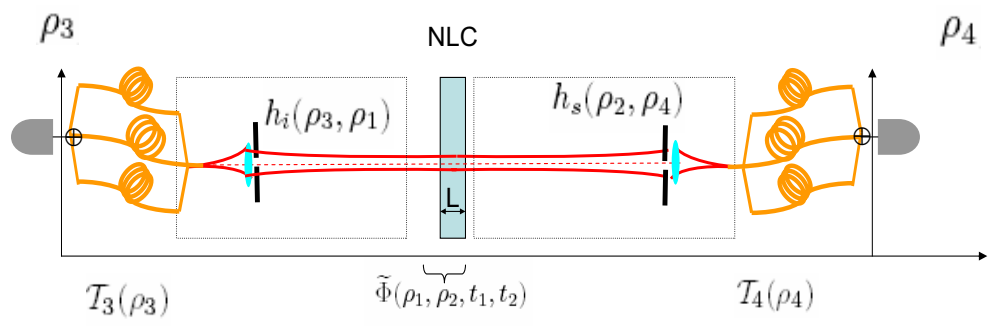

Figure 3. Unfolded picture of an object corresponding to the idler channel of a PDC source, generated in a nonlinear crystal NLC of length $L$. The object (on the left) is an incoherent source prepared by a multi-mode fiber, and the image is collected by a multimode fiber.

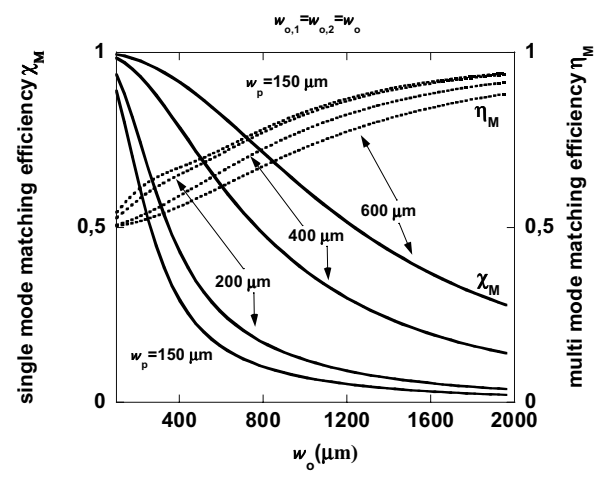

Figure 4. Plots of $\eta_{M}$ (dashed) and $\chi_{M}$ (solid) for $w_{o, 1}=w_{o, 2}=w_{o}$ (solid lines) and various pump waists, $w_{p}=$ $150,200,400,600 \mu \mathrm{m}$, versus $w_{o}$.

with thin crystal limit

$$
\eta_{P}=\frac{w_{o, 1}^{2} w_{o, 2}^{2}\left(w_{o, 1}^{2}+w_{p}^{2}\right)}{w_{o, 2}^{2} w_{p}^{2}+w_{o, 1}^{2}\left(w_{o, 2}^{2}+w_{p}^{2}\right)}
$$

In the multi-mode approach presented here, the preparation and collection modes can be thought of as spatially filtering or selecting the multi-mode input light. As Fig.4 demonstrates, the predictions made by this model yield different results than the single-mode model. The multi-mode model predicts that, for a fixed pump waist, the maximum multi mode-matching efficiency is obtained when the fiber-defined collection mode (at the 


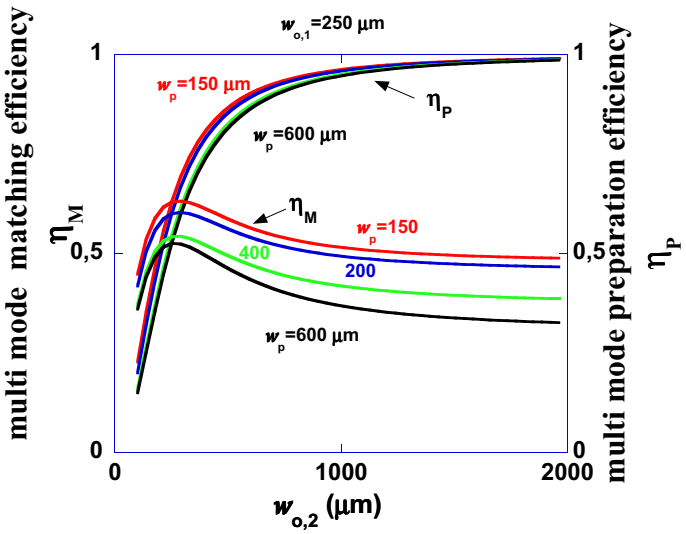

Figure 5. Plot of $\eta_{p, M}$ versus the collecting mode waist for various pump waist, $w_{p}=150,200,400,600 \mu \mathrm{m}$ and for fixed preparing waist $w_{o, 1}=250 \mu \mathrm{m}$.

crystal) is large, i.e. all the pumped crystal volume is in a region of unit collection efficiency of the spatial filter system. With the single-mode model, one of the fibers acts as a source of a single-mode beam that propagates through a spatial filter (in this case the pumped crystal volume) to the other fiber. The maximum mode-matching efficiency is achieved with a large pump waist, with respect to the preparation and collection beam waist at the crystal. If the pump waist is smaller than the fiber-defined collection beam waist, the mode-matching efficiency is reduced. In this case, the efficiency is maximized with $w_{o, 1}=w_{o, 2}=w_{o}$, but this presents the practical difficulty of having, and aligning, exactly the same selected modes. Here, the differences between the two models are more evident. In fact, when the collection/preparation waist is much greater than the pump waist, $\eta_{M}$ asymptotically goes to 1 , while $\chi_{M}$ goes to 0 ; in the opposite condition $\left(w_{p} \gg w_{o}\right), \eta_{M} \rightarrow \frac{1}{2}$ and $\chi_{M} \rightarrow 1$.

In Fig.5, we plot the theoretical prediction of $\eta_{P, M}$ for a $5 \mathrm{~mm}$ crystal of $\mathrm{LiIO}_{3}$ versus the collecting waist $w_{o, 2}$ for fixed preparing waist $w_{o, 1}=250 \mu \mathrm{m}$ at the crystal with a range of pump waists. As it appears for $\eta_{p}$ there is not an optimum collecting waist and the highest efficiency is reached faster for the smaller pump waist. The $\eta_{M}$ presents instead a finite optimum waist for smaller pump waist, but the highest efficiency is never reached, because modes are matched only on average, given the statistical nature of the multimode model. 


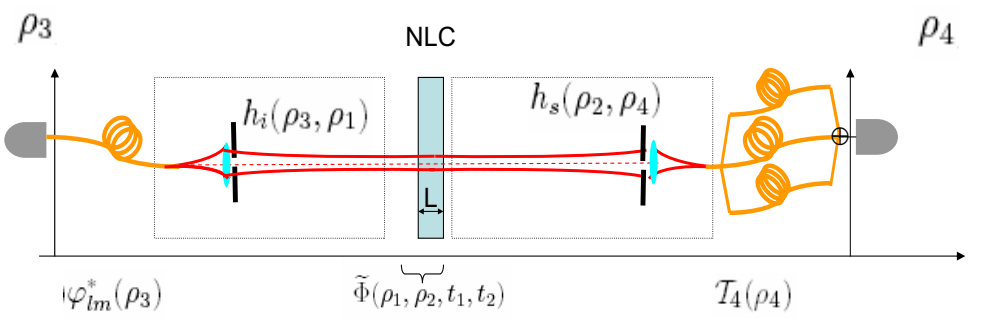

Figure 6. Unfolded scheme for the preparation of the heralding channel in a single mode, while photons are collected in a bucket detection scheme by an aperture or a multi-mode fiber placed in the focus of a lens

\section{SINGLE MODE PREPARATION AND MULTIMODE COLLECTION EFFICIENCY}

When we prepare the source according the unfolded scheme of Fig.6 in a single mode, while we collect in a multi-mode, the single counts are $\mathcal{C}_{3}$ in Eq.(3), and the coincidences are:

$$
\mathrm{C}_{34}=\int \mathrm{d} t_{1} \mathrm{~d} t_{2} \mathrm{~d}^{2} \rho_{4} \mathcal{T}\left(\rho_{4}\right)\left|\int \mathrm{d}^{2} \rho_{1} \mathrm{~d}^{2} \rho_{2} \mathrm{~d}^{2} \rho_{3} \widetilde{\Phi}_{12}\left(\rho_{1}, t_{1}, \rho_{2}, t_{2}\right) h_{i}\left(\rho_{1}, \rho_{3}\right) h_{s}\left(\rho_{2}, \rho_{4}\right) \varphi_{l m}^{*}\left(\rho_{3}\right)\right|^{2}
$$

The single-mode preparation and multi-mode collection efficiency is

$$
\epsilon_{P}=\frac{\mathrm{C}_{34}}{\mathcal{C}_{3}}
$$

We explicitly calculate it with a mode preparation given by a single-mode fiber in a perfect imaging configuration (lens with infinite aperture and magnification $M_{3}$ arranged to place the collection beam waist, $w_{o, 1}$ at the crystal), described as a Gaussian field, $\varphi_{10}\left(\rho_{3}\right)$, while collecting in a multi-mode with a Gaussian aperture $\mathcal{T}_{4}\left(\rho_{4}\right)=e^{-\frac{2 \rho_{4}^{2}}{w^{2}}}$. The signal optical system is $\left.h_{s}\left(\rho_{2}, \rho_{4}\right)=\exp \left[-i k\left(\rho_{2}-\rho_{4}\right)^{2}\right)\right]$, the free space propagation, with $k=\pi /\left(\lambda_{s} d\right)$ and $d$ the propagation distance. The single mode preparation and multi-mode collection efficiency is given by

$$
\epsilon_{P}=\mathcal{F}_{P}^{\prime} \frac{\left(-\mathcal{N}_{p}+\mathcal{N}_{s}+K_{p} \theta_{i}\right) k^{2} w^{2} w_{o, 1}^{2} w_{p}^{2} \sqrt{w_{o, 1}^{2}+w_{p}^{2}}}{\sqrt{C^{\prime} B^{\prime}}},
$$

with

$$
\mathcal{F}^{\prime}{ }_{P}=\frac{\operatorname{Erf}\left[\frac{L \sqrt{2 B^{\prime}}}{K_{p} \sqrt{C^{\prime}}}\right]}{\operatorname{Erf}\left[\sqrt{2} L \frac{\left(-\mathcal{N}_{p}+\mathcal{N}_{s}+K_{p} \theta_{i}\right)}{K_{p} \sqrt{w_{o, 1}^{2}+w_{p}^{2}}}\right]},
$$




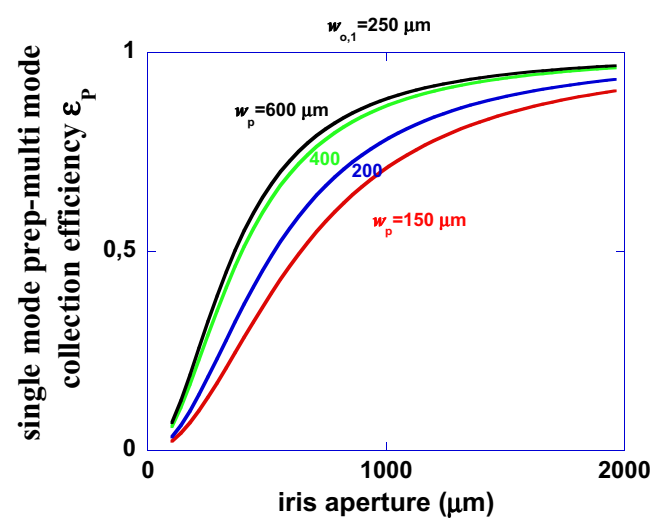

Figure 7. Plot of $\epsilon_{P}$ versus the collecting iris aperture for various pump waist, $w_{p}=150,200,400,600 \mu \mathrm{m}$

$B^{\prime}=\left(\mathcal{N}_{p}-\mathcal{N}_{s}+K_{p} \theta_{s}\right)^{2} w_{o, 1}^{4} w_{p}^{2} k^{2}+\left(-\mathcal{N}_{p}+\mathcal{N}_{s}+K_{p} \theta_{i}\right)^{2}\left(w_{o, 1}^{2}+w_{p}^{2}+k^{2} w^{2} w_{o, 1}^{2} w_{p}^{2}\right)+K_{p}^{2} k^{2} w_{o, 1}^{2} w_{p}^{4}\left(\theta_{i}+\theta_{s}\right)^{2}$, and $C^{\prime}=\left(w_{o, 1}^{2}+w_{p}^{2}\right)^{2}+k^{2} w_{o, 1}^{2} w_{p}^{2}\left(w^{2} w_{p}^{2}+w_{o, 1}^{2} w^{2}+w_{o, 1}^{2} w_{p}^{2}\right)$. In the thin crystal limit this reduces to

$$
\epsilon_{P}=\frac{k^{2} w^{2} w_{o, 1}^{2} w_{p}^{2}\left(w_{o, 1}^{2}+w_{p}^{2}\right)}{\left(w_{o, 1}^{2}+w_{p}^{2}\right)^{2}+k^{2} w_{o, 1}^{2} w_{p}^{2}\left(w^{2} w_{p}^{2}+w_{o, 1}^{2} w^{2}+w_{o, 1}^{2} w_{p}^{2}\right)}
$$

We observed that in this case $\epsilon_{P}$ depends slightly on $L$. We plot in Fig.7, the theoretical prediction of $\epsilon_{P}$ for a $5 \mathrm{~mm}$ crystal of $\mathrm{LiIO}_{3}$ versus the iris aperture $w$ for fixed preparing waist $w_{o, 1}=250 \mu \mathrm{m}$ at the crystal, for different pump waist configurations. In this case we have an intermediate behavior between $\chi_{P}$ and $\eta_{P}$, the highest efficiency is obtained faster for larger pump waist. We underline that the comparison with the following experimental results show a qualitative analogy with this curve, because the $k$ parameter plays an essential rule in the estimate of the efficiency. Specifically the quantitative value of $k$ in the simulation does not correspond to the experimental values.

\section{PRELIMINARY EXPERIMENTAL RESULTS}

We give here some preliminary experimental results and compare them with theoretical predictions, showing the agreement. The simulations and measurements are done exciting PDC in a type I phase-matching configuration with a pump wavelength of $351 \mathrm{~nm}$ and by a $\mathrm{LiIO}_{3}$ crystal length of $5 \mathrm{~mm}$. Pump waists at the crystal were ranging from from $150 \mu \mathrm{m}$ to $600 \mu \mathrm{m}$.

Some measurements were done with the trigger arm (heralding channel) coupled with a single-mode fiber. The lens images the minimum waist at the crystal. The signal arm was either coupled with a single-mode fiber in 

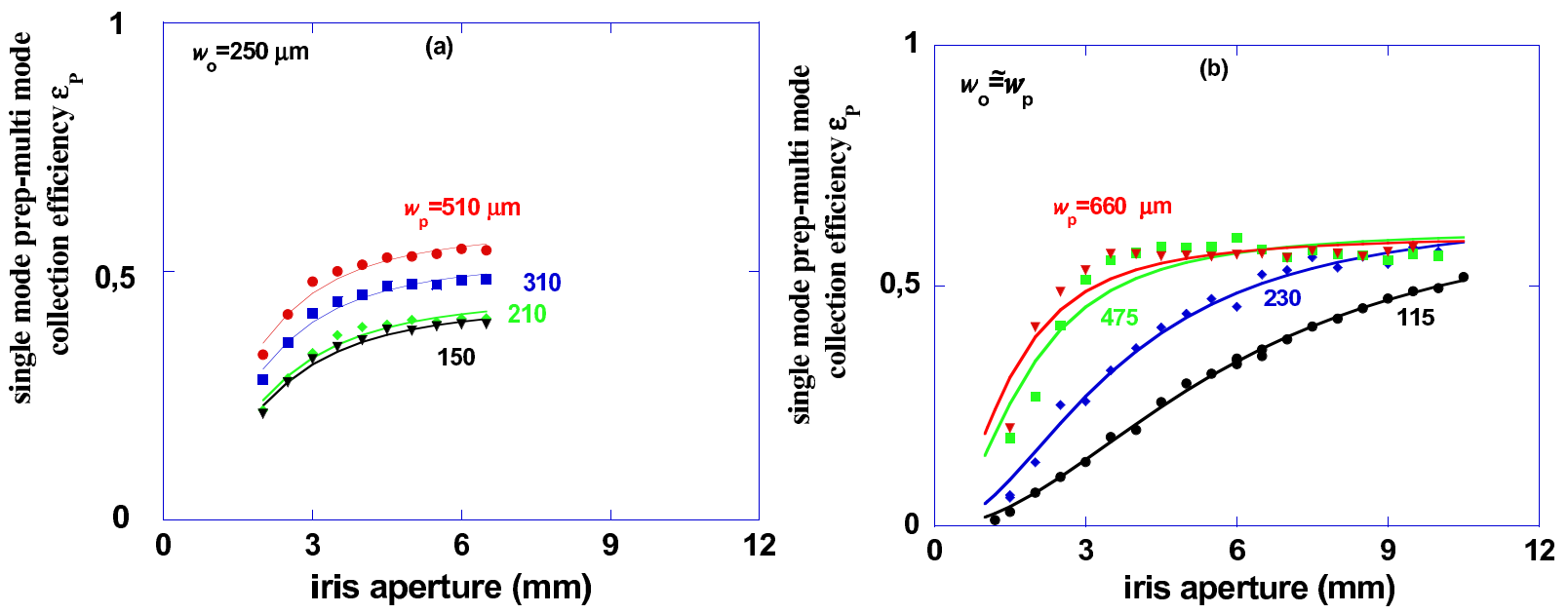

Figure 8. Plots of $\epsilon_{P}$ data versus the iris diameter (a) for a range of pump waist with fixed $w_{o}=250 \mu \mathrm{m}$ and (b) for a range of pump waists with $w_{p} \approx w_{o}$. (a) shows measurements performed at IEN, where the efficiency is uncorrected for the detector dead-time, while (b) shows measurements performed at NIST, corrected for dead-time. The solid line are fits obtained with the expected theoretical curve in the thin-crystal limit reported in Eq.(24).

a perfect imaging configuration or with a multimode-fiber placed in the focus of the coupling lens, where an iris at the lens selects the collecting modes. Other measurements were performed in a similar setup $\left(6 \mathrm{~mm} \mathrm{LiIO}_{3}\right)$ at the National Institute of Standards and Technology (NIST) with the trigger beam coupled with a single-mode fiber and the signal coupled to the detector by a single lens, with an iris selecting the modes. In particular two experimental configurations were chosen. In one configuration the trigger mode was fixed to maximize the singles rate, while in the other configuration the heralding channel waist was matched to the pump waist at the crystal.

In Fig. 8 we show the experimental values of the single-mode preparation and multi-mode collection efficiency $\left(\epsilon_{P}\right)$ versus the collecting iris diameter, for the two experimental configurations, i.e. for fixed $w_{o}=250 \mu \mathrm{m}$ and (b) for fixed $w_{p} \approx w_{o}$ and a range of pump waists as indicated. In both cases the maximum efficiency is obtained for larger pump waists as predicted by the theory in Fig. 7 .

We point out that the theoretical prediction of the multi-mode collection and multi mode preparation efficiency $\left(\eta_{P}\right)$ versus the equivalent Gaussian filter collection aperture $w_{o, 2}$ would instead give the maximum efficiency for smaller pump waists. However, while the single-mode preparation and matching efficiency $\left(\chi_{M, P}\right)$ versus the collecting waist $w_{o, 2}$ present a maximum for properly matching the three modes, $\eta_{P, M}$ may not reach the highest efficiency even in this case.

Fig. 9 shows the experimental single counts from a heralding channel coupled with a single-mode fiber versus the fiber waist at the crystal, for various pump waists. Data are fitted by theoretical curves that for single mode coupling are $\mathcal{C}_{3}=\frac{K_{p} \operatorname{Erf}\left[\frac{L \sqrt{2}\left(-N_{p}+N_{s}+K_{p} \theta_{i}\right)}{L K_{p}\left(\sqrt{w_{o}^{2}+w_{p}^{2}}\right)}\right]}{\sqrt{2 \pi}\left(-N_{p}+N_{s}+K_{p} \theta_{i}\right)\left(\sqrt{w_{o}^{2}+w_{p}^{2}}\right)}$; here the data clearly matches the single-mode propagation because we have an optimum preparation waist that maximizes singles rate (note that the maximum singles rate are for a smaller pump waist). In a multi-mode configuration the single rates would just increase with the iris or filter aperture. In Fig. 10 we plot the experimental data of the uncorrected efficiency in the case of single mode

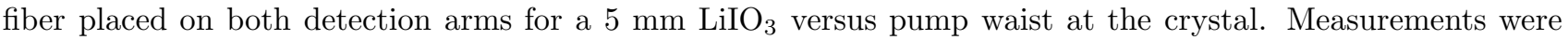
performed for two different preparing and collecting mode waists configuration, that is the apparent waists at the crystal were $w_{o, 1}=110 \mu \mathrm{m}$ and $w_{o, 2}=50 \mu \mathrm{m}$ and reversed. The data are fitted by the Eq.(11) and Eq.(12), in the thin crystal configuration, this is because of the very small size of the waists, where the theory for long crystal is no longer reliable. The agreement shows the correctness of model proposed. 


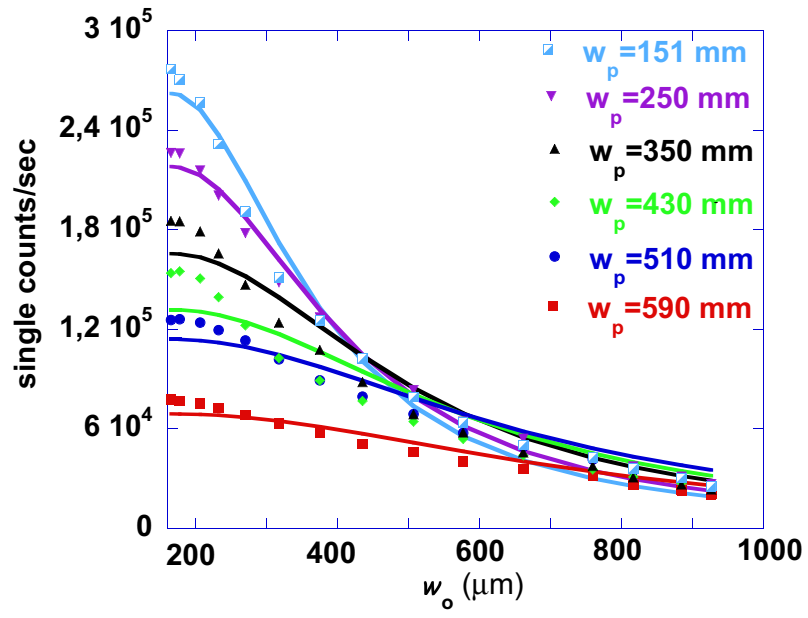

Figure 9. Plots of single counts $\mathcal{C}_{3}$ versus $w_{o}$ for a range of pump waists. The maximum counts are for the smallest pump waist. 


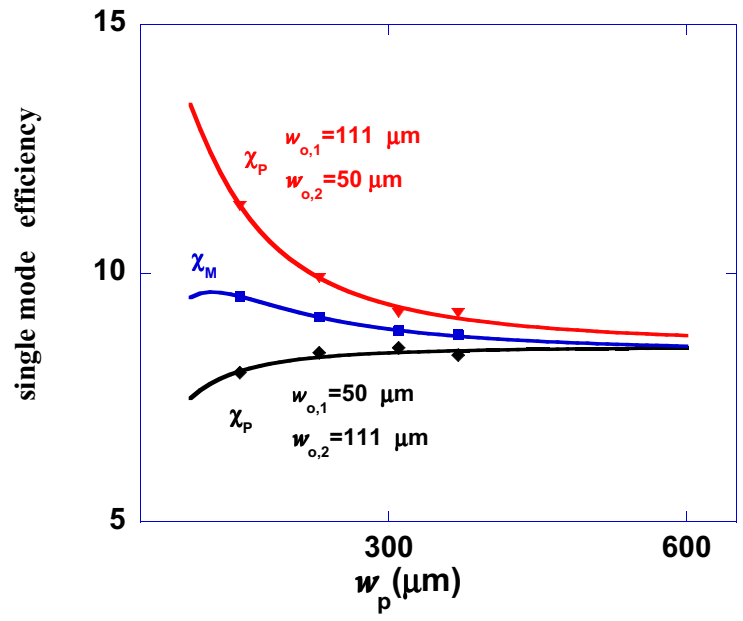

Figure 10. Plots of the single mode preparation efficiency $\chi_{P}$ and matching efficiency $\chi_{M}$ versus the pump waist $w_{p}$ for two preparing and collecting mode configurations, $w_{o, 1}=111 \mu \mathrm{m}$ and $w_{o, 2}=50 \mu \mathrm{m}$ the upper curve and $w_{o, 1}=50 \mu \mathrm{m}$ and $w_{o, 2}=111 \mu \mathrm{m}$ the lower curve. The curve in the middle is $\chi_{M}$ for $w_{o, 1}=155 \mu \mathrm{m}$ and $w_{o, 2}=70 \mu$. The solid lines are fit obtained by the proposed theoretical model. 


\section{CONCLUSIONS}

In summary, we have presented an analytic model to quantify the mode preparation and matching efficiency in terms of adjustable experimental parameters with the goal of optimizing single-mode collection from PDC sources. In addition, we have presented an alternative scheme that may have more validity for multi-mode collection arrangements. We reported preliminary experimental results, supporting the validity of the proposed multi-mode collection model.

\section{ACKNOWLEDGMENTS}

This work was supported in part by DARPA/QUIST, ARDA, ARO.

\section{REFERENCES}

1. A. L. Migdall, S. Castelletto, I. P. Degiovanni, and M. L. Rastello, "Intercomparison of a correlated-photonbased method to measure detector quantum efficiency," Applied Optics 41(11), pp. 2914-2922, 2002.

2. W. Tittel, J. Brendel, H. Zbinden, and N. Gisin, "Quantum cryptography using entangled photons in energy-time bell states," Physical Review Letters 84(20), pp. 4737-4740, 2000.

3. E. Knill, R. Laflamme, and G. J. Milburn, "A scheme for efficient quantum computation with linear optics," Nature 409, pp. 46-52, 2001.

4. T. B. Pittman, B. C. Jacobs, and J. D. Franson, "Single photons on pseudo-demand from stored parametric down-conversion," quant-ph/0205103v1, 2002.

5. A. Migdall, D. Branning, and S. Castelletto, "Tailoring single-photon and multiphoton probabilities of a single-photon on-demand source," Phys. Rev. A 66, p. 053805, 2002.

6. M. H. Rubin, "Transverse correlation in optical spontaneous parametric down-conversion," Phys. Rev. A 54(6), pp. 5349-5360, 1996.

7. S. Castelletto, I. P. Degiovanni, A. L. Migdall, and M. Ware, "On the measurement of two-photon singlemode coupling efficiency in parametric downconversion photon sources," New Journal of Physics , 2004.

8. D. N. Klyshko, "Two-photon light: Influence of filtration and new possible EPR experiment," Physics Letters A 128(3), pp. 133-137, 1988.

9. F. A. Bovino, P. Varisco, A. M. Colla, G. Castagnoli, G. Di Giuseppe, and A. V. Sergienko, "Effective fiber coupling of entangled photons for quantum communication," Optics Communication 227, 2003.

10. S. Castelletto, I. P. Degiovanni, M. Ware, and A. L. Migdall, "Coupling efficiency in single photon on demand sources," in Proceedings SPIE Quantum Information and Quantum Imaging, 5161, pp. 48-56, 2003.

11. C. Monken, P. H. Souto Ribeiro, and S. Padua, "Optimizing the photon pair collection effeciency: A step towards a loophole-free Bell's inequalities experiment," Phys. Rev. A 57, pp. R2267-R2269, 1998. 